\title{
Golgi and Related Vesicle Proteomics: Simplify to Identify
}

\author{
Joan Gannon, John J.M. Bergeron, and Tommy Nilsson
}

The Research Institute of the McGill University Health Centre and the Department of Medicine, McGill University, Montreal, Quebec H3A 1A1, Canada

Correspondence: tommy.nilsson@mcgill.ca

\begin{abstract}
Despite more than six decades of successful Golgi research, the fundamental question as to how biosynthetic material is transported through the secretory pathway remains unanswered. New technologies such as live cell imaging and correlative microscopy have highlighted the plastic nature of the Golgi, one that is sensitive to perturbation yet highly efficient in regaining both structure and function. Single molecule-microscopy and super resolution-microscopy further adds to this picture. Various models for protein transport have been put forward, each with its own merits and pitfalls but we are far from resolving whether one is more correct than the other. As such, our laboratory considers multiple mechanisms of Golgi transport until proven otherwise. This includes the two classical modes of transport, vesicular transport and cisternal progression/maturation as well as more recent models such as tubular inter- and intra-cisternal connections (long lasting or transient) and inter-Golgi stack transport. In this article, we focus on an emerging inductive technology, mass spectrometry-based proteomics that has already enabled insight into the relative composition of compartments and subcompartments of the secretory pathway including mechanistic aspects of protein transport. We note that proteomics, as with any other technology, is not a stand-alone technology but one that works best alongside complementary approaches.
\end{abstract}

\section{PROTEOMICS AS A TECHNOLOGY}

A described and thoroughly discussed in a
mass spectrometry (MS)-based proteomics as
currently deployed is correlative despite its
high mass accuracy. Yet it allows for a compre-
hensive and quantitative characterization of
the protein composition of entire organelles,
substructures, protein complexes, and bio-
chemical fractions (e.g., detergent fractions
upon phase separation). In a typical proteomic study, protein samples are separated according to their size using gel electrophoresis, i.e., Polyacrylamide Gel Electrophoresis or PAGE (e.g., SDS-PAGE) or by both size and isoelectric point, i.e., 2-D PAGE. Gel fragments containing separated proteins are then subjected to protease digestion in-gel, usually with trypsin, to generate peptides that are then subjected to liquid chromatography (LC). From the LC column, these peptides then enter the mass spectrometer where they are subjected to a first or parental MS scan. Using this parental scan,

Editors: Graham Warren and James Rothman

Additional Perspectives on The Golgi available at www.cshperspectives.org

Copyright (C) 2011 Cold Spring Harbor Laboratory Press; all rights reserved; doi: 10.1101/cshperspect.a005421

Cite this article as Cold Spring Harb Perspect Biol 2011;3:a005421 
J. Gannon et al.

either all the peaks or a selection can be subjected to fragmentation followed by another MS scan (termed tandem mass spectrometry [MS/MS] and when combined with LC, LC-MS/MS). The molecular weights (MWs) of observed peptides and their fragmented counterparts are calculated and then compiled and parsed with a database or databases using a search engine such as Mascot. Simplistically, peptides of predicted open reading frames are generated in silico and used to match the MS-derived data. By correlating calculated MWs with predicted ones, and combining this with peptide sequence coverage, they can be identified. Using the relative abundance of the redundant peptides identified for each protein, they can be quantitated. The correlative aspect allows identification at a probability level that is usually $95 \%$ or higher. This number can be set empirically as a trade-off between the rate of false positives and that of assumed correct positives in which the former can be gauged by deploying a database where each open reading frame has been reversed. Using this reference database, it is possible to set search parameters such that confidence in the end is $95 \%$ or higher. Nevertheless, protein identification may still be ambiguous even at a $95 \%$ or higher confidence as databases are full of erroneous annotations and multiple redundant entries that necessitate manual curation. These erroneous annotations include subcellular localization showing mitochondrial proteins in the nucleus, ER chaperones in the plasma membrane and so forth. Proteomics-based publications are therefore full of proteins in new and exciting locations usually without attempts to back up such data through independent validation (for example, by immunofluorescence). This has contributed to the wariness that cell biologists and others have of (MS)-based proteomics.

\section{LIMITATIONS OF PROTEOMICS}

The biggest limitation of (MS)-based proteomics as applied today is its limited dynamic range. Typically, MS instruments yield only 3-4 orders of magnitude dynamic range. Practically, using plasma as an example, one would need 6-8 orders of magnitude as plasma levels of serum albumin and interleukin 6 (IL6) differ by 6 orders of magnitude in their respective concentrations, albumin being the more prominent one. To increase the dynamic range, it is possible to pre-enrich the sample through subcellular fractionation prior to analysis. As covered in a previous review (Bergeron et al. 2010), the distinction is made between purifying an organelle to apparent homogeneity or to correlate relative sedimentation behavior under various conditions. With quantitative (MS)-based proteomics, the two can be combined such that the desired dynamic range is achieved as well as enabling correlation of uncharacterized proteins with known marker proteins as envisaged by de Duve (De Duve 1964). Indeed, using intracellular membranes enriched through fractionation, it is possible to track simultaneously thousands of proteins across gradients. Uncharacterized/novel proteins of a compartment or substructure can then be localized by their ability to codistribute with known marker proteins of a particular organelle or substructure. Using bioinformatics tools (e.g., principal component analysis [PCA] and hierarchal clustering algorithms), proteins can then be grouped together with those that display a similar sedimentary behavior. This can easily be extended to biochemical properties as revealed through detergent solubility, detergent partitioning, salt resistance, and so on. Using this approach, which we term the protein microscope (Bell et al. 2007), it is feasible to tease out function through "association." Protein contaminants from undesired structures are also revealed by this approach, as these show no correlation. We are currently combining this approach with high-resolution native gel electrophoresis following gradient fractionation (Dejgaard et al. 2010). This combination of methods enables physical native protein complexes to be isolated and characterized quantitatively. This technology is readily applicable today and ought to be of interest to cell biologists and those from associated fields as an alternative to coimmunoprecipitation or Fluorescence Resonance Energy Transfer (FRET) microscopy. With this article, we wish 
Golgi Proteomics: Simplify to Identify

to demonstrate that (MS)-based proteomics can be a powerful tool by focusing on a longstanding question as to the composition of intra-Golgi transport carriers termed COPI vesicles and how these relate to transport of newly synthesized proteins through the secretory pathway.

\section{SETTING THE STAGE FOR STUDYING THE CONTENT OF COPI TRANSPORT VESICLES}

Two models of protein transport through the secretory pathway have dominated the Golgi field for more than five decades; the cisternal maturation/progression model (Grasse 1957) (CMPM) and the vesicular transport model (Jamieson and Palade 1967) (VTM). Early electron microscopy (EM) studies had shown that Golgi stacks consist of flattened cisternae stacked close together and connected laterally, cisterna to cisterna, via highly fenestrated regions. Approximately 100-150 such stacks are joined together laterally to yield the Golgi ribbon (the Golgi). Each Golgi stack also has fenestrations on the cis- and the trans-side and this gave rise to an early notion that membranes would assemble at the cis-side and disassemble at the trans-side. Cisternal ER membranes, filled with biosynthetic material, were thought to break off from the ER and align themselves with previously detached ER-derived cisternae giving rise to the stacked appearance of the Golgi. Hence, the CMPM, as originally postulated, depended on a constant input of membranes and biosynthetic material from the ER. This was disproved by Palade and Jamieson (Jamieson and Palade 1968) who showed that intracellular transport and biosynthesis of proteins are independent of each other. When protein synthesis was inhibited; there was no discernable loss of Golgi stacks or apparent changes in morphology and the CMPM was therefore largely abandoned.

The CMPM was replaced by the VTM, which depicts small 50- to 70-nm vesicles as transport carriers of biosynthetic material from the ER to the Golgi and from cisterna to cisterna within the Golgi stack (intra-Golgi transport). In the 1980s and the 1990s, the molecular and functional framework of transport vesicles were successfully delineated through yeast genetics, EM, and biochemistry (Rothman and Fine 1980; Novick et al. 1981; Orci et al. 1986; Barlowe et al. 1994). These studies identified both COPII and COPI vesicles as responsible, respectively, for transporting biosynthetic material out of the ER, and for moving this from cisterna to cisterna. Much of this success was based on the use of an in vitro transport assay that monitors transfer of $\mathrm{N}$-acetylglucosamine onto the $\mathrm{N}$-linked oligosaccharide of a temperature sensitive mutant of the $G$ protein of vesicular stomatitis virus (VSV- $\left.\mathrm{G}_{\mathrm{ts} 045}\right)$. The temperature-sensitive $G$ protein allowed for a pulse-like release from the ER with a subsequent positioning in the Golgi stack upon transfer to ice. When performed in $\mathrm{CHO}$ cells deficient in N-acetylglucosaminyltransferase I (GlcNAcT1), this yielded Golgi stacks suitable as donors for transport carriers (e.g., COPI vesicles). During the 1980s and the 1990s, much of the emphasis was placed on this in vitro transport assay, monitoring the transfer of VSV-G from donor to acceptor Golgi. This assay built on previous studies of transport of viral proteins through the secretory pathway at a time where most viewed Golgi stacks as stable membrane entities, compartments, populated by specific sets of processing enzymes (e.g., glycosylation enzymes).

It was then assumed that through cisternal compartmentalization, Golgi-resident glycosylation enzymes, and other processing enzymes could act in an orderly manner on the biosynthetic material as it moved from cisterna to cisterna via COPI vesicles. Biochemical fractionation experiments and immuno-EM supported this view. Glycosylation enzymes acting on the N-linked high mannose form were localized to the early part of the pathway (e.g., the cis-Golgi network and the cis-cisternae) whereas enzymes acting on $\mathrm{N}$-linked complex oligosaccharides were found later in the pathway (trans-Golgi and the trans-Golgi network) (Roth and Berger 1982; Dunphy et al. 1985). The notion of strictly compartmentalized Golgi-resident glycosylation enzymes was challenged in the mid-1990s through double 
immuno-EM studies showing that glycosylation enzymes distributed across the Golgi stack in gradient-like distributions (Nilsson et al. 1993; Rabouille et al. 1995). Here, classical medial and a trans enzyme were shown to occupy the same cisterna. There were arguments as to the validity of summing up gold particles over multiple stacks, which was subsequently addressed using quantitative photo-oxidation of EGFP tagged to N-acetylgalactosaminyltransferase 2 (GalNAc-T2) revealing a gradient-like distribution across a single stack (Grabenbauer et al. 2005). Such enzyme distributions pointed to a more dynamic organization of Golgi-resident glycosylation enzymes within the Golgi apparatus and opened up for the possibility of continuous recycling to offset the flow of biosynthetic material (see Saraste and Kuismanen [1992] for a modified CMPM).

At that time, a mechanistic framework for recycling had already been established for proteins that reside in the ER and in the interface between the ER and the Golgi apparatus. Both soluble (Munro and Pelham 1987) and transmembrane (Nilsson et al. 1989) constituents were shown to display discrete sorting signals that are sufficient to ensure the return proteins from the Golgi apparatus to the ER. Both operate through COPI vesicles as shown by Cosson and Letourneur who discovered that coatomer, a 7 subunit coat complex, binds directly to the cytoplasmic ER/Golgi sorting signal $\mathrm{K}(\mathrm{X}) \mathrm{KK}-\mathrm{COOH}$ showing that $\mathrm{COPI}$ vesicles, at least in part, are involved in retrograde transport (Cosson and Letourneur 1994; Letourneur et al. 1994). The question as to whether COPI vesicles could also be involved in retrograde transport of Golgi resident glycosylation enzymes from later parts of the Golgi stack (medial and trans cisternae) remained open. At the end of the 90s, two parallel in vitro studies suggested that indeed, COPI vesicles were able to transfer GlcNAc-T1 activity using the transport assay described above and that they did so efficiently (Lanoix et al. 1999; Love et al. 1998). Fractions enriched in smaller membranes consistent with vesicles were shown to contain most of the GlcNAc-T1 needed for glycosylation of VSV-G, the latter confined to larger membrane structures (e.g., cisternae) (Cosson and Letourneur 1994). In a subsequent study it was shown that formation of the small membrane structures were sensitive to brefeldin A, a fungal metabolite, that inhibits COPI vesicle formation and stimulates tubule formation (Lin et al. 1999). At the same time, an independent study using vesicles formed in vitro using highly purified rat liver Golgi fractions showed that these contained glycosylation enzymes at higher concentrations than those found in the Golgi cisternae (Lanoix et al. 1999). A corresponding depletion of biosynthetic material into such vesicles was also observed. Importantly, such vesicles were by EM morphologically consistent with COPI vesicles, required coatomer, ADP-ribosylation factor 1 (ARF1), energy and cytosol to form, and were functional as deduced by their ability to efficiently transport GlcNAc-T1 in the in vitro transport assay described above.

Incorporation and concentration of glycosylation enzymes into COPI vesicles were shown to require hydrolysis of GTP by ARF1 suggesting an active and selective sorting event (Lanoix et al. 1999). This explained why, up until that time, COPI vesicles studied by EM and biochemistry had appeared empty of resident glycosylation enzymes as these were usually purified in the presence of nonhydrolysable GTP, GTP $\gamma$ S. Without GTP hydrolysis, sorting is inhibited, rendering the interpretation of analyzed COPI vesicles difficult. A subsequent study showed that there is more than one population of COPI vesicles that predominantly contain resident Golgi proteins (Lanoix et al. 2001), however, this does not exclude subpopulations also involved in anterograde transport (Pelham and Rothman 2000). That anterograde subpopulations of COPI vesicles exist is supported by a study that used different tethering factors to capture COPI vesicles followed by western blotting to indicate that vesicles with marker proteins of biosynthetic proteins were different from those with resident proteins thus suggesting that both anterograde and retrograde COPI transport vesicles coexist (Malsam et al. 2005). Attempts were made to complement the above in vitro studies by 
examining the distribution of biosynthetic material as well as Golgi resident enzymes by immuno-EM in cell lines. Whereas the highly abundant biosynthetic cargo protein albumin could be shown to be restricted to Golgi cisternae (Dahan et al. 1994; Martinez-Menarguez et al. 1999) the distribution of mannosidase II (Mann II) in NRK cells was inconclusive as two labs, prominent in their respective proficiency in EM, came to completely opposite conclusions as to the incorporation of Mann II into COPI buds and peri-Golgi vesicles (MartinezMenarguez et al. 2001; Cosson et al. 2002). Both studies used the same antibody and the same cell type, NRK cells, clearly pointing to a limitation of immuno-EM as a technique when examining the content of small vesicular structures such as COPI vesicles. Arguments as to the validity of the data obtained with in vitro formed vesicles were also put forward based on earlier studies (Quinn et al. 1984; Takamori et al. 2006; Dupuy and Engelman 2008) indicating that the protein concentration of transmembrane proteins in the Golgi cisternae was already very high. Therefore, further sorting/concentration through ARF regulated GTP hydrolysis seemed unlikely (Pelham and Rothman 2000). In other words, transmembrane proteins were so highly concentrated in Golgi cisternae they were not expected to concentrate further in COPI vesicles. A new approach was needed, one that could quantitatively compare the entire protein content of COPI vesicles with that of Golgi cisternae.

\section{USING PROTEOMICS TO DETERMINE THE CONTENT OF COPI VESICLES}

A large-scale protocol was developed for the formation of COPI vesicles using highly purified rat liver Golgi membranes ( $>100$ times over homogenate) as a source material, rat liver cytosol, an ATP regenerating system and a recombinant dominant negative mutant of $\alpha$-SNAP to prevent fusion of formed vesicles. As a control, vesicles were also generated in vitro in the presence of GTP $\gamma$ S. Following purification of vesicles through gradient sedimentation followed by gradient floatation, vesicles were extensively characterized by western blotting and electron microscopy, including morphometry and immuno-labeling, the latter using antibodies directed to the cytoplasmic domains of p24 proteins of early Golgi cisternae. Vesicle fractions were then subjected to solubilization and separation by SDS-PAGE followed by quantitative LC-MS/MS. The protein content of these formed vesicles was also compared to the other membranes of the secretory pathway, smooth and rough endoplasmic reticulum, as well as Golgi cisternae. The resulting MS data were then used to search a nonredundant rat database to yield peptide and protein identifications. Protein identification required extensive manual curation involving manual BLAST steps to ensure that annotated peptides did indeed correspond to the correct protein (more than one peptide of a protein is usually observed making peptide assignment and protein identification feasible). Quantification was performed through redundant peptide counting enabling fractions to be compared through PCA. This analysis showed that the protein composition of each structure analyzed differed significantly. This was important as it had previously been suggested that COPI vesicles generated in vitro represent Golgi fragments rather than bona fide COPI vesicles (Kweon et al. 2004). PCA without any functional assignments of proteins showed that analyzed COPI vesicles were clearly distinct entities compared to Golgi cisternae. The control, vesicles generated in the presence of GTP $\gamma \mathrm{S}$, were also found to be distinct from COPI vesicles and Golgi cisternal membranes (Gilchrist et al. 2006). PCA was followed by hierarchical clustering in order to group proteins according to their relative enrichment/de-enrichment (abundance) across the different fractions analyzed. As validation, the degree of coclustering of the seven subunits of coatomer, the coat complex of COPI vesicles, was monitored. As coatomer is a highly stable cytosolic complex attaching to both cisternal membranes as well as vesicles in a GTP-specific manner, these would be predicted to cocluster. Six subunits were found to be tightly coclustered with the seventh subunit adjacent to this cluster demonstrating the 
J. Gannon et al.

robustness of the approach taken. It is here important to realize that coatomer present in the different fractions was solubilized and individual chains separated by SDS-PAGE followed by in-gel trypsin digest and analysis by LC-MS / MS in the presence of thousands of other peptides derived from other proteins present in the fraction. The ability to reconstruct the coatomer complex, based purely on relative enrichment-de-enrichment, validates, in our opinion, the coclustering approach we term the protein microscope. With the PCA revealing that each membrane component analyzed is distinct, it was possible to ask which proteins contribute to this. A simple criterion was used to highlight all proteins that were enriched in COPI vesicles over that of the Golgi cisternae. Importantly, all proteins with known functions were, without exception, found to be enriched in COPI vesicles and these were Golgi resident proteins. This showed that en masse, COPI vesicles generated from purified Golgi membranes, in vitro, preferentially incorporate resident constituents of the secretory pathway rather than biosynthetic materials. Also, that such preferential incorporation requires hydrolysis of GTP (by ARF/ARFGAP [Lanoix et al. 1999]) as control vesicles generated in the presence of GTP $\gamma S$ revealed no such enrichment. With vesicles generated under permissive GTP hydrolysis conditions, there was more than a twofold enrichment of Golgi resident proteins. Using the protein density observed in synaptic vesicles (Takamori et al. 2006), there is theoretically room for at least a three-fold concentration of membrane proteins in Golgi cisternae (Nilsson et al. 2009). This makes it possible for COPI vesicles to concentrate Golgi-resident proteins. This points to COPI vesicles as having a major role in recycling at all levels of the secretory pathway, consistent with earlier findings showing that proteins endowed with a luminal KDEL sequence can recycle via the KDEL receptor all the way back from the TGN (Miesenbock and Rothman 1995).

Whether a twofold enrichment is sufficient to ensure that all Golgi resident proteins can be recycled in the context of a CMPM remains to be seen. We presently know very little in terms of the half-life of COPI vesicles, how quickly they form, and how fast they are consumed through fusion with the adjacent cisterna. It is therefore impossible to determine the relative contribution of COPI vesicles to overall transport vis-à-vis the CMPM or the VTM or other scenarios. Also, and as noted above, our findings do not exclude possible subpopulations of anterograde transport vesicles or the additional roles of transient tubules and direct inter cisternal continuities in these transport events. We do know that membrane proteins along with lipids may also recycle from the TGN to the ER via a COPI-independent pathway, which interestingly, is the preferred route taken by certain toxins to gain access to the ER (Girod et al. 1999). Isolation and LC-MS/MS characterization of COPI vesicles coupled with in vivobased microscopy should yield more insight into the nature of COPI vesicles and how these relate to the overall question of protein transport in the Golgi apparatus.

\section{USING PROTEOMICS AND THE PROTEIN MICROSCOPE TO IDENTIFY AND CHARACTERIZE NEW GOLGI PROTEINS}

Of the approximately 20,300 proteins predicted by the human genome and a similar number in rodents, more than half have little or no documented function. About a thousand or more have never been observed at all by any method. When proteins of unknown function are uncovered, whether indirectly via cDNA cloning, high throughput microscopy-based screens or more recently through tandem mass spectrometry, they are often proved to be biologically relevant. If these "novel" proteins are linked to an organelle then it is the major function of the organelle that can give the greatest insight into protein function. Calnexin, for example, was purified from the ER as an integral membrane phosphoprotein. The primary sequence was deduced from Edman degradation of derived peptide sequences that allowed selection of primers for cDNA cloning. This information rapidly led to its function as lectin/chaperone. In turn, this led to the uncovering of a glycan-based code for glycoprotein folding, a process which 
ensures the spatial temporal coordination of productive folding and presentation to the ER exit machinery through the calnexin cycle (Schrag et al. 2003). Calnexin also retains misfolded glycoproteins in the ER to present them to the ER associated degradation machinery (Chevet et al. 2010). Protein folding and quality control proteins are the most abundant resident proteins of the ER (Gilchrist et al. 2006).

A similar trajectory of discovery has been followed for the novel Golgi protein first uncovered by tandem mass spectrometry and originally named GPP34 (Golgi peripheral protein of $34 \mathrm{kDa}$ ) and published online in October 2000 (Bell et al. 2001). The tandem mass spectrometry identified unambiguously tryptic peptide sequences that did not match any known protein in the protein database but did in the EST database. The full-length sequence was deduced from EST sequences, as were those of predicted orthologs in several species including yeast and human. By analytical fractionation and immune labeling at the LM and EM level the protein was localized to the stacked cisternae of the Golgi apparatus. Wu et al. independently identified this same protein by proteomic analysis of isolated Golgi apparatus, naming it GMX33 (Golgi matrix protein of $33 \mathrm{kDa}$ ) with its full-length sequence deduced by cDNA sequencing. In budding yeast the gene is nonessential but complemented by the mammalian gene ( $\mathrm{Tu}$ et al. 2008; Corbacho et al. 2010). The annotation in yeast is VPS74 (Vacuolar protein sorting-associated protein 74) due to an effect on sorting of proteins to the vacuole. The X-ray structure of VPS74 suggests an association with the short amino terminus stub of the type II integral membrane proteins of Golgi located sugar transferases (Schmitz et al. 2008). A postulated direct association of VPS74 with the cytosolic domain of Golgi located sugar transferases ( $\mathrm{Tu}$ et al. 2008) seems very surprising since mammalian GPP34 complements yeast VPS74 but no sequence similarity is known for yeast and mammalian Golgi located sugar transferases.

Despite this caveat much more is now understood for mammalian GPP34. The human ortholog (known as GOLPH3-golgi phosphoprotein 3) was uncovered as the first example of a Golgi located oncoprotein (Scott et al. 2009). This astonishing observation was a consequence of a genome wide array based on a comparative hybridization study of 307 solid tumors from nonsmall cell lung carcinoma, ovarian carcinoma, prostate cancer, and melanoma to screen for amplified genes casually linked to these tumors. GPP34/GOLPH3 was found when overexpressed to transform mouse embryo fibroblasts and confer anchorage independent growth in immortalized human melanocytes. Overexpression also led to the activation of the mTOR pathway and was associated with cell size regulation. Melanoma tumors overexpressing GPP34/GOLPH3 were highly aggressive in immune deficient mice with the mTOR pathway activiated. Not surprisingly, rapamycin treatment inhibited the TOR pathway and markedly diminished the tumors.

More recently, GPP34 has been identified as the major Golgi protein associated with the Golgi located phospholipid phosphtidyl inositol 4-phospate in mammals (Dippold et al. 2009) and yeast (Wood et al. 2009). In mammalian cells, Dippold et al. have shown that GPP34 may also be responsible for the Golgi ribbon; the structure that links different stacked Golgi cisternae into one continuum. It has been postulated that the flattened cisternae themselves are maintained by an association of GPP34/GOLPH3 with myosin 18a connected to F-actin.

There were more than 70 proteins of unknown function uncovered by proteomics of highly purified Golgi fractions (Gilchrist et al. 2006). By applying the protein microscope, these unknown proteins are seen to codistribute with proteins of known function (Gilchrist et al. 2006). Since Golgi membranes and COPI vesicles were also compared with both rough and smooth ER, it was possible to highlight ER proteins in their respective compartment through hierarchical clustering. Through this, a new function was uncovered for ERp44 (endoplasmic reticulum resident protein 44), which belongs to the family of protein disulfide isomerases that have evolved 
to catalyze disulfide bond formation coincident with productive protein folding. Functional and localization studies using ERp44 EGFP fusion proteins had established this member as an ER resident protein along with its client proteins (Anelli et al. 2002, 2003). Studies also showed that ERp44 has a role in the ER retention of ERO1 (Bertoli et al. 2004; Qiang et al. 2007); the inositol trisphosphate (IP3) receptor (Tateishi et al. 2005) and the secretory cargo protein adiponectin (Wang et al. 2007). Rather than belonging to the ER, however, we found this that protein, through proteomics, to be almost exclusively localized to the Golgi apparatus, a postulate confirmed by indirect immunofluorescence using an antibody to the endogenous protein (Gilchrist et al. 2006). In fact, ERp44 was found concentrated about 14-fold in Golgi membranes compared with ER membranes. The question immediately arose as to how a predominant Golgi location could be reconciled with a function of ERp44 in protein folding and the retention of ER membrane proteins and soluble secretory proteins. The answer found in the spatial and temporal requirements of protein maturation where ERp44 acts as a retention protein for ERO1, the IP3 receptor and adiponectin, retrieving these proteins as they escape from the ER. For adiponectin, its regulated secretion in fat cells is a consequence of a signaling pathway that abrogates its recycling via ERp44 (Wang et al. 2007; Wang et al. 2008; Wolf 2008; Phillips et al. 2009; Long et al. 2010). Importantly, client proteins that do not associate via cysteine residue oxidation to the thioredoxin motifs of ERp44 include the formylglycine-activating enzyme (Mariappan et al. 2008). This protein must be presented to a Golgi located ERp44, which then returns it to the ER and presents it to sulfatases to catalyze formation of formylglycine. This is crucial for function and so far, all known mutations in humans that cause multiple sulfatase deficiency are those of the formylglycine activating enzyme that only affect the transport of this enzyme to the Golgi apparatus (Fraldi et al. 2008; Dierks et al. 2009). If unable to first associate with the Golgi localized ERp44, transport defective mutants of formylglycine activating enzyme are unable to recognize the sulfatases preventing their maturation into active enzymes (Mariappan et al. 2008).

\section{CONCLUDING REMARKS}

Despite technical caveats and challenges currently facing MS-based proteomics, its application in cell biology has already proven fruitful. Insights into the composition of a COPI vesicle generated in vitro has extended their role in recycling to all levels of the secretory pathway. Most notably, COPI vesicles transport Golgi resident glycosylation enzymes. Further work examining the composition of subpopulations of COPI vesicles should test for the possibility of vesicles predominantly carrying biosynthetic cargo, presumably in the anterograde direction.

\section{REFERENCES}

Anelli T, Alessio M, Mezghrani A, Simmen T, Talamo F, Bachi A, Sitia R. 2002. ERp44, a novel endoplasmic reticulum folding assistant of the thioredoxin family. EMBO J 21: $835-844$.

Anelli T, Alessio M, Bachi A, Bergamelli L, Bertoli G, Camerini S, Mezghrani A, Ruffato E, Simmen T, Sitia R. 2003. Thiol-mediated protein retention in the endoplasmic reticulum: The role of ERp44. EMBO J 22: 5015-5022.

Barlowe C, Orci L, Yeung T, Hosobuchi M, Hamamoto S, Salama N, Rexach MF, Ravazzola M, Amherdt M, Schekman R. 1994. COPII: A membrane coat formed by Sec proteins that drive vesicle budding from the endoplasmic reticulum. Cell 77: 895-907.

Bell AW, Ward MA, Blackstock WP, Freeman HN, Choudhary JS, Lewis AP, Chotai D, Fazel A, Gushue JN, Paiement J, et al. 2001. Proteomics characterization of abundant Golgi membrane proteins. J Biol Chem 276: 5152-5165.

Bell AW, Nilsson T, Kearney RE, Bergeron JJ. 2007. The protein microscope: Incorporating mass spectrometry into cell biology. Nature methods 4: 783-784.

Bergeron JJ, Au CE, Desjardins M, McPherson PS, Nilsson T. 2010. Cell biology through proteomics-ad astra per alia porci. Trends Cell Biol 20: 337-345.

Bertoli G, Simmen T, Anelli T, Molteni SN, Fesce R, Sitia R. 2004. Two conserved cysteine triads in human Erol $\alpha$ cooperate for efficient disulfide bond formation in the endoplasmic reticulum. J Biol Chem 279: 3004730052.

Chevet E, Smirle J, Cameron PH, Thomas DY, Bergeron JJ. 2010. Calnexin phosphorylation: Linking cytoplasmic signalling to endoplasmic reticulum lumenal functions. Semin Cell Dev Biol 21: 486-490. 
Corbacho I, Olivero I, Hernandez LM. 2010. Identification of the MNN3 gene of Saccharomyces cerevisiae. Glycobiology 20: 1336-1340.

Cosson P, Letourneur F. 1994. Coatomer interaction with di-lysine endoplasmic reticulum retention motifs. Science 263: $1629-1631$.

Cosson P, Amherdt M, Rothman JE, Orci L. 2002. A resident Golgi protein is excluded from peri-Golgi vesicles in NRK cells. Proc Natl Acad Sci 99: 12831-12834.

Dahan S, Ahluwalia JP, Wong L, Posner BI, Bergeron JJ. 1994. Concentration of intracellular hepatic apolipoprotein $\mathrm{E}$ in Golgi apparatus saccular distensions and endosomes. J Cell Biol 127: 1859-1869.

De Duve C. 1964. Principles of tissue fractionation. J Theoret Biol 6: 33-59.

Dejgaard K, Theberge JF, Heath-Engel H, Chevet E, Tremblay ML, Thomas DY. 2010. Organization of the Sec61 translocon, studied by high resolution native electrophoresis. J Proteome Res 9: 1763-1771.

Dierks T, Schlotawa L, Frese MA, Radhakrishnan K, von Figura K, Schmidt B. 2009. Molecular basis of multiple sulfatase deficiency, mucolipidosis II/III and NiemannPick C1 disease-Lysosomal storage disorders caused by defects of non-lysosomal proteins. Biochim Biophys Acta 1793: 710-725.

Dippold HC, Ng MM, Farber-Katz SE, Lee SK, Kerr ML, Peterman MC, Sim R, Wiharto PA, Galbraith KA, Madhavarapu S, et al. 2009. GOLPH3 bridges phosphatidylinositol-4-phosphate and actomyosin to stretch and shape the Golgi to promote budding. Cell 139: 337-351.

Dunphy WG, Brands R, Rothman JE. 1985. Attachment of terminal $\mathrm{N}$-acetylglucosamine to asparagine-linked oligosaccharides occurs in central cisternae of the Golgi stack. Cell 40: 463-472.

Dupuy AD, Engelman DM. 2008. Protein area occupancy at the center of the red blood cell membrane. Proc Natl Acad Sci 105: 2848-2852.

Fraldi A, Zito E, Annunziata F, Lombardi A, Cozzolino M, Monti M, Spampanato C, Ballabio A, Pucci P, Sitia R, et al. 2008. Multistep, sequential control of the trafficking and function of the multiple sulfatase deficiency gene product, SUMF1 by PDI, ERGIC-53 and ERp44. Hum Mol Genet 17: 2610-2621.

Gilchrist A, Au CE, Hiding J, Bell AW, Fernandez-Rodriguez J, Lesimple S, Nagaya H, Roy L, Gosline SJ, Hallett M, et al. 2006. Quantitative proteomics analysis of the secretory pathway. Cell 127: 1265-1281.

Girod A, Storrie B, Simpson JC, Johannes L, Goud B, Roberts LM, Lord JM, Nilsson T, Pepperkok R. 1999. Evidence for a COP-I-independent transport route from the Golgi complex to the endoplasmic reticulum. Nat Cell Biol 1: 423-430.

Grabenbauer M, Geerts WJ, Fernadez-Rodriguez J, Hoenger A, Koster AJ, Nilsson T. 2005. Correlative microscopy and electron tomography of GFP through photooxidation. Nat Method 2: 857-862.

Grasse PP. 1957. Ultrastructure, polarity and reproduction of Golgi apparatus. CR Hebd Seances Acad Sci 245: $1278-1281$.

Jamieson JD, Palade GE. 1967. Intracellular transport of secretory proteins in the pancreatic exocrine cell. I. Role of the peripheral elements of the Golgi complex. J Cell Biol 34: 577-596.

Jamieson JD, Palade GE. 1968. Intracellular transport of secretory proteins in the pancreatic exocrine cell. 3. Dissociation of intracellular transport from protein synthesis. J Cell Biol 39: 580-588.

Kweon HS, Beznoussenko GV, Micaroni M, Polishchuk RS, Trucco A, Martella O, Di Giandomenico D, Marra P, Fusella A, Di Pentima A, et al. 2004. Golgi enzymes are enriched in perforated zones of golgi cisternae but are depleted in COPI vesicles. Mol Biol Cell 15: 4710-4724.

Lanoix J, Ouwendijk J, Lin CC, Stark A, Love HD, Ostermann J, Nilsson T. 1999. GTP hydrolysis by arf-1 mediates sorting and concentration of Golgi resident enzymes into functional COP I vesicles. $E M B O J \mathbf{1 8}$ : 4935-4948.

Lanoix J, Ouwendijk J, Stark A, Szafer E, Cassel D, Dejgaard K, Weiss M, Nilsson T. 2001. Sorting of Golgi resident proteins into different subpopulations of COPI vesicles: A role for ArfGAP1. J Cell Biol 155: 1199-1212.

Letourneur F, Gaynor EC, Hennecke S, Demolliere C, Duden R, Emr SD, Riezman H, Cosson P. 1994. Coatomer is essential for retrieval of dilysine-tagged proteins to the endoplasmic reticulum. Cell 79: 1199-1207.

Lin CC, Love HD, Gushue JN, Bergeron JJ, Ostermann J. 1999. ER/Golgi intermediates acquire Golgi enzymes by brefeldin A-sensitive retrograde transport in vitro. J Cell Biol 147: 1457-1472.

Long Q, Lei T, Feng B, Yin C, Jin D, Wu Y, Zhu X, Chen X, Gan L, Yang Z. 2010. Peroxisome proliferator-activated receptor- $\gamma$ increases adiponectin secretion via transcriptional repression of endoplasmic reticulum chaperone protein ERp44. Endocrinology 151: 3195-3203.

Love HD, Lin CC, Short CS, Ostermann J. 1998. Isolation of functional Golgi-derived vesicles with a possible role in retrograde transport. J Cell Biol 140: 541-551.

Malsam J, Satoh A, Pelletier L, Warren G. 2005. Golgin tethers define subpopulations of COPI vesicles. Science 307: 1095-1098.

Mariappan M, Radhakrishnan K, Dierks T, Schmidt B, von Figura K. 2008. ERp44 mediates a thiol-independent retention of formylglycine-generating enzyme in the endoplasmic reticulum. J Biol Chem 283: 6375-6383.

Martinez-Menarguez JA, Geuze HJ, Slot JW, Klumperman J. 1999. Vesicular tubular clusters between the ER and Golgi mediate concentration of soluble secretory proteins by exclusion from COPI-coated vesicles. Cell 98: 81-90.

Martinez-Menarguez JA, Prekeris R, Oorschot VM, Scheller R, Slot JW, Geuze HJ, Klumperman J. 2001. Peri-Golgi vesicles contain retrograde but not anterograde proteins consistent with the cisternal progression model of intraGolgi transport. J Cell Biol 155: 1213-1224.

Miesenbock G, Rothman JE. 1995. The capacity to retrieve escaped ER proteins extends to the trans-most cisterna of the Golgi stack. J Cell Biol 129: 309-319.

Munro S, Pelham HR. 1987. A C-terminal signal prevents secretion of luminal ER proteins. Cell 48: 899-907.

Nilsson T, Jackson M, Peterson PA. 1989. Short cytoplasmic sequences serve as retention signals for transmembrane proteins in the endoplasmic reticulum. Cell 58: 707-718. 
J. Gannon et al.

Nilsson T, Pypaert M, Hoe MH, Slusarewicz P, Berger EG, Warren G. 1993. Overlapping distribution of two glycosyltransferases in the Golgi apparatus of HeLa cells. $J$ Cell Biol 120: 5-13.

Nilsson T, Au CE, Bergeron JJ. 2009. Sorting out glycosylation enzymes in the Golgi apparatus. FEBS Lett 583: 3764-3769.

Nilsson T, Mann M, Aebersold R, Yates JR III, Bairoch A, Bergeron JJ. 2010. Mass spectrometry in high-throughput proteomics: Ready for the big time. Nat Method 7: 681-685.

Novick P, Ferro S, Schekman R. 1981. Order of events in the yeast secretory pathway. Cell 25: 461-469.

Orci L, Glick BS, Rothman JE. 1986. A new type of coated vesicular carrier that appears not to contain clathrin: Its possible role in protein transport within the Golgi stack. Cell 46: $171-184$.

Pelham HR, Rothman JE. 2000. The debate about transport in the Golgi-two sides of the same coin? Cell 102: 713-719.

Phillips SA, Kung J, Ciaraldi TP, Choe C, Christiansen L, Mudaliar S, Henry RR. 2009. Selective regulation of cellular and secreted multimeric adiponectin by antidiabetic therapies in humans. Am J Physiol Endocrinol Metab 297: E767-E773.

Qiang L, Wang H, Farmer SR. 2007. Adiponectin secretion is regulated by SIRT1 and the endoplasmic reticulum oxidoreductase Ero1-L $\alpha$. Mol Cell Biol 27: 4698-4707.

Quinn P, Griffiths G, Warren G. 1984. Density of newly synthesized plasma membrane proteins in intracellular membranes II. Biochemical studies. J Cell Biol 98: $2142-2147$.

Rabouille C, Hui N, Hunte F, Kieckbusch R, Berger EG, Warren G, Nilsson T. 1995. Mapping the distribution of Golg enzymes involved in the construction of complex oligosaccharides. J Cell Sci 108: 1617-1627.

Roth J, Berger EG. 1982. Immunocytochemical localization of galactosyltransferase in HeLa cells: Codistribution with thiamine pyrophosphatase in trans-Golgi cisternae. J Cell Biol 93: 223-229.

Rothman JE, Fine RE. 1980. Coated vesicles transport newly synthesized membrane glycoproteins from endoplasmic reticulum to plasma membrane in two successive stages. Proc Natl Acad Sci 77: 780-784.
Saraste J, Kuismanen E. 1992. Pathways of protein sorting and membrane traffic between the rough endoplasmic reticulum and the Golgi complex. Seminars Cell Biol 3: $343-355$.

Schmitz KR, Liu J, Li S, Setty TG, Wood CS, Burd CG, Ferguson KM. 2008. Golgi localization of glycosyltransferases requires a Vps74p oligomer. Dev Cell 14: 523-534.

Schrag JD, Procopio DO, Cygler M, Thomas DY, Bergeron JJ. 2003. Lectin control of protein folding and sorting in the secretory pathway. Trends Biochem Sci 28: 49-57.

Scott KL, Kabbarah O, Liang MC, Ivanova E, Anagnostou V, Wu J, Dhakal S, Wu M, Chen S, Feinberg T, et al. 2009. GOLPH3 modulates mTOR signalling and rapamycin sensitivity in cancer. Nature 459: 1085-1090.

Takamori S, Holt M, Stenius K, Lemke EA, Gronborg M, Riedel D, Urlaub H, Schenck S, Brugger B, Ringler P, et al. 2006. Molecular anatomy of a trafficking organelle. Cell 127: 831-846.

Tateishi Y, Hattori M, Nakayama T, Iwai M, Bannai H, Nakamura T, Michikawa T, Inoue T, Mikoshiba K. 2005. Cluster formation of inositol 1,4,5-trisphosphate receptor requires its transition to open state. J Biol Chem 280: 6816-6822.

Tu L, Tai WC, Chen L, Banfield DK. 2008. Signal-mediated dynamic retention of glycosyltransferases in the Golgi. Science 321: 404-407.

Wang ZV, Schraw TD, Kim JY, Khan T, Rajala MW, Follenzi A, Scherer PE. 2007. Secretion of the adipocyte-specific secretory protein adiponectin critically depends on thiol-mediated protein retention. Mol Cell Biol 27: 3716-3731.

Wang Y, Lam KS, Yau MH, Xu A. 2008. Post-translational modifications of adiponectin: Mechanisms and functional implications. Biochem J 409: 623-633.

Wolf G. 2008. New insights into thiol-mediated regulation of adiponectin secretion. Nutr Rev 66: 642-645.

Wood CS, Schmitz KR, Bessman NJ, Setty TG, Ferguson KM, Burd CG. 2009. PtdIns4P recognition by Vps74/ GOLPH3 links PtdIns 4-kinase signaling to retrograde Golgi trafficking. J Cell Biol 187: 967-975.

Wu CC, Taylor RS, Lane DR, Ladinsky MS, Weisz JA, Howell KE. 2000. GMx33: A novel family of trans-Golgi proteins identified by proteomics. Traffic 1: 963-975. 


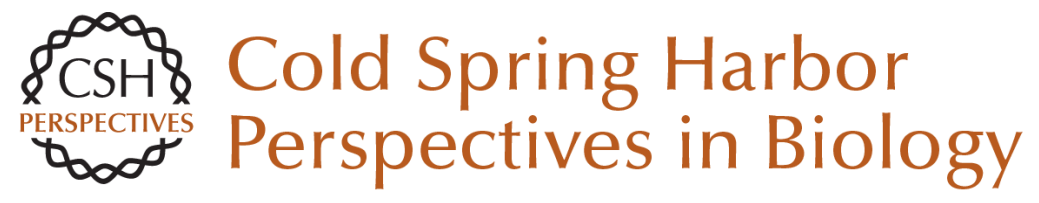

\section{Golgi and Related Vesicle Proteomics: Simplify to Identify}

Joan Gannon, John J.M. Bergeron and Tommy Nilsson

Cold Spring Harb Perspect Biol 2011; doi: 10.1101/cshperspect.a005421 originally published online August 2, 2011

\section{Subject Collection The Golgi}

Structure of Golgi Transport Proteins

Daniel Kümmel and Karin M. Reinisch

\section{Golgi Biogenesis}

Yanzhuang Wang and Joachim Seemann

Golgi Glycosylation and Human Inherited

Diseases

Hudson H. Freeze and Bobby G. Ng

Models for Golgi Traffic: A Critical Assessment

Benjamin S. Glick and Alberto Luini

\section{Architecture of the Mammalian Golgi} Judith Klumperman

Evolution and Diversity of the Golgi Mary J. Klute, Paul Melançon and Joel B. Dacks

Evolutionary Forces Shaping the Golgi

Glycosylation Machinery: Why Cell Surface

Glycans Are Universal to Living Cells Ajit Varki

Golgi Positioning

Smita Yadav and Adam D. Linstedt
Golgi and Related Vesicle Proteomics: Simplify to Identify Joan Gannon, John J.M. Bergeron and Tommy Nilsson

Organization of SNAREs within the Golgi Stack Jörg Malsam and Thomas H. Söllner

Golgi during Development Weimin Zhong

Entry and Exit Mechanisms at the cis-Face of the Golgi Complex Andrés Lorente-Rodríguez and Charles Barlowe

COPI Budding within the Golgi Stack Vincent Popoff, Frank Adolf, Britta Brügger, et al.

Mechanisms of Protein Retention in the Golgi David K. Banfield

The Golgin Coiled-Coil Proteins of the Golgi

Apparatus Sean Munro

Signaling at the Golgi Peter Mayinger

For additional articles in this collection, see http://cshperspectives.cshlp.org/cgi/collection/

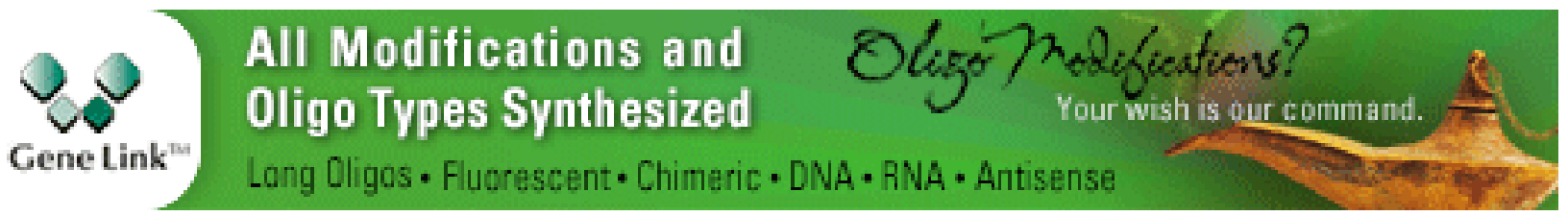

